\title{
\begin{tabular}{l|l} 
pcori)? & PATIENT-CENTERED OUTCOMES RESEARCH INSTITUTE \\
RESEARCH SUMMARY
\end{tabular}
}

PROJECT INFORMATION

March 2020

\section{Developing New Statistical Methods for Clinical Studies Where Patients Choose Their Treatments}

Principal investigator

Organization

Denise Esserman, MS, PhD

Yale University

\section{What was the project about?}

A patient's preference for a treatment may affect how well the treatment works. For example, if patients prefer a specific medicine, they may be more likely to take that medicine.

Traditional randomized clinical trials can't tell how much patient preferences affect how well a treatment works. But a two-stage clinical trial might. In a twostage trial, researchers assign patients by chance to one of two groups. In the first group, researchers assign patients by chance to get a specific treatment, regardless of their preference. In the second group, patients choose their treatment. In a two-stage trial, researchers can compare health outcomes for patients who choose their treatment with patients who don't. But few methods exist for researchers to design and analyze this type of trial.

In this project, the research team developed new statistical methods for two-stage trials. The team wanted to find out how many patients are needed for two-stage trials to provide accurate results. They also wanted to learn how to measure whether patient preference for a specific treatment affects patients' health outcomes.

\section{What did the research team do?}

The research team developed statistical methods to figure out how patient treatment preferences affect different types of health outcomes. One method can be used with the types of outcomes that could be true or false, such as having or not having a symptom.
Another method was for outcomes that can be counted, such as the number of symptoms a patient had. The team also developed a formula to calculate the number of patients to include in the study.

The research team created test data using a computer to check how the methods worked. The test data looked like data from two-stage trials. The team also tested the methods using data from two example trials.

The research team worked with other researchers and statisticians to develop and test the methods.

\section{What were the results?}

The new methods worked well to figure out how many patients to include in a two-stage trial, how well treatments worked, and how patient preferences affected health outcomes. The methods worked in studies with many or few patients. The methods also worked in the example two-stage trials. Studies needed to include more patients when different groups of patients, such as men and women, have different treatment preferences.

\section{What were the limits of the project?}

The research team didn't try all possible ways of testing the methods using the computer program. The methods may not work well if many patients are unsure of which treatment they want.

Future research could develop other methods for analyzing results of two-stage trials. 


\section{How can people use the results?}

Researchers can use these new methods to design two-stage trials and learn how patient treatment preferences affect how well treatments work.
To learn more about this project, visit www.pcori.org/Esserman309. 